DAMTP-2000-44

CERN-TH/2000-200

IFIC/00-34

UNIL-IPT/00-11

July 2000

\title{
Primordial spectrum of gauge fields from inflation
}

\author{
Anne-Christine Davis, ${ }^{1}$ Konstantinos Dimopoulos, ${ }^{2}$ Tomislav Prokopec, ${ }^{3}$ \\ and \\ Ola Törnkvist ${ }^{1,4}$ \\ ${ }^{1}$ Department of Applied Mathematics and Theoretical Physics, University of Cambridge, \\ Wilberforce Road, Cambridge CB3 0WA, United Kingdom \\ ${ }^{2}$ Instituto de Física Corpuscular, Universitat de Valencia/CSIC, \\ Apartado de Correos 2085, 46071 Valencia, Spain \\ ${ }^{3}$ Université de Lausanne, Institut de Physique Théorique, \\ BSP, CH-1015 Lausanne, Switzerland \\ ${ }^{4}$ CERN Theory Division, 1211 Genève 23, Switzerland
}

\begin{abstract}
We show that conformal invariance of gauge fields is naturally broken in inflation, having as a consequence amplification of gauge fields. The resulting spectrum of the field strength is approximately $B_{\ell} \propto \ell^{-1}$, where $\ell$ is the relevant coherence scale. One realisation of our scenario is scalar electrodynamics with a scalar whose mass is large enough to evade observational constraints - the obvious candidates being supersymmetric partners of the standard-model fermions. Our mechanism also leads naturally to amplification of the standard-model $Z$ boson field due to its coupling to the electroweak Higgs field. At preheating, the spectrum of the $Z$ field is transferred to the hypercharge field, which remains frozen in the plasma and is converted into a magnetic field at the electroweak phase transition. With a reasonable model of field evolution one obtains a magnetic field strength of the order of $10^{-29}$ Gauss on a scale of $100 \mathrm{pc}$, the size of the largest turbulent eddy in a virialised galaxy. Resonant amplification in preheating can lead to primordial fields as large as $10^{-24}$ Gauss, consistent with the seed field required for the galactic dynamo mechanism.

${ }^{1}$ acd@damtp.cam.ac.uk, ${ }^{2}$ kostas@flamenco.ific.uv.es

${ }^{3}$ Tomislav.Prokopec@ipt.unil.ch, ${ }^{4}$ o.tornkvist@damtp.cam.ac.uk
\end{abstract}




\section{Introduction}

It is well known that during inflation the inflaton, being a light scalar field, couples gravitationally and grows on superhorizon scales. Likewise, (scalar) cosmological perturbations grow resulting in a scale-invariant spectrum on superhorizon scales, providing one of the most important predictions of inflation: seeds for formation of large-scale structures in the Universe [1, 2]. The spectrum of gravitational waves generated during inflation has also been studied in detail [1] and is regarded as perhaps the only signature of inflation that may be "observed" directly. It is hence surprising that the evolution of vector fields has not been considered with sufficient care, especially in light of the exciting possibility that primordial gauge fields from inflation could produce the magnetic fields observed today in galaxies [3] and even affect the cosmic microwave background radiation [4].

It is usually assumed that gauge fields do not grow during inflation. The reason is very simple: gauge fields do not couple gravitationally to a conformally flat space-time. The metric of conformally flat space-times can in general be written as $g_{\mu \nu}=a^{2}(\tau, \vec{x}) \eta_{\mu \nu}$, where $\eta_{\mu \nu}=\operatorname{diag}[1,-1,-1,-1]$ is the Minkowski metric. The space-times of standard cosmology are all conformally flat. Indeed, in inflationary de Sitter space-time the scale factor reads $a(\tau)=-1 /\left(H_{\mathrm{I}} \tau\right)$, where $H_{\mathrm{I}}$ is the Hubble parameter in inflation; in the radiation era $a \propto \tau$, while in the matter era $a \propto \tau^{2}$. This implies that, in order to get any amplification of gauge fields during inflation, conformal invariance must be broken.

Various proposals have been put forward in which conformal invariance of gauge fields is broken. A particularly nice one is due to Dolgov [5], who showed that the conformal anomaly of the gauge-field stress-energy tensor, expressed by the triangle diagram, leads to particle production for gauge fields even in a conformally flat space-time. Turner and Widrow [6] consider several ways of breaking conformal invariance: (a) gravitational coupling of the photon, (b) anomalous coupling of the photon to the axion, and (c) coupling of the photon to a charged, massless scalar field. In the former two cases, conformal anomaly is broken by a non-standard coupling of the photon field. The case (c) has recently been explored by Calzetta et al. and by Kandus et al. [7]. These authors argue that, as a consequence of scalar

charge separation during inflation, charged domains form which proceed to source electric currents in the radiation era, leading to magnetic-field production. Their claim, that the magnetic field thus created has sufficient strength to seed the galactic dynamo mechanism 
[8], has recently been contested by Giovannini and Shaposhnikov [9].

In this Letter we show that there is a natural way of breaking conformal invariance of gauge fields which has not been considered in the literature. Namely, the backreaction of a charged scalar field gives the gauge field an effective mass, which breaks conformal invariance.

\section{Scalar electrodynamics}

We begin by considering scalar electrodynamics, which nicely illustrates our mechanism of gauge-field amplification from inflation. The idea can be easily extended to include nonAbelian gauge fields and multi-component scalar fields. The Lagrangian is

$$
\mathcal{L}_{\phi \mathrm{ED}}=-\frac{1}{4} g^{\mu \eta} g^{\nu \rho} F_{\mu \nu} F_{\eta \rho}+g^{\mu \nu}\left(D_{\mu} \phi\right)^{\dagger} D_{\nu} \phi-m_{\phi}^{2} \phi^{\dagger} \phi-\lambda_{\phi}\left(\phi^{\dagger} \phi\right)^{2},
$$

where $D_{\mu}=\partial_{\mu}-i e A_{\mu}$ is the covariant derivative, $F_{\mu \nu}=\partial_{\mu} A_{\nu}-\partial_{\nu} A_{\mu}$ is the gauge field strength, $g^{\mu \nu}=a^{-2} \eta^{\mu \nu}$ is a conformally flat metric, and $m_{\phi}$ and $\lambda_{\phi}$ are the mass and self-coupling of the scalar field $\phi$, respectively. The experimental constraint on the $\phi$ mass, $m_{\phi} \gg M_{\mathrm{EW}} \simeq 100 \mathrm{GeV}$, is easily satisfied. The obvious candidates for $\phi$ are supersymmetric partners of the standard-model leptons and quarks. In addition, we require that $m_{\phi} \ll H_{\mathrm{I}}$, so that the charged scalar field may grow during inflation.

The relevant equation of motion for the photon field is obtained from Eq. (1) as the Lagrange equation $\partial_{\nu}\left(\delta_{\partial_{\nu} A_{\mu}}\left(\sqrt{-D_{g}} \mathcal{L}_{\phi \mathrm{ED}}\right)\right)-\delta_{A_{\mu}}\left(\sqrt{-D_{g}} \mathcal{L}_{\phi \mathrm{ED}}\right)=0$, where $D_{g}=\operatorname{det}\left[g_{\mu \nu}\right]=$ $-a^{8}$. After some algebra one finds that the mode equation for the transverse component of the photon field in the Hartree approximation and in unitary gauge can be recast as

$$
\left(\partial_{\tau}^{2}+\vec{k}^{2}+e^{2} a^{2}\left\langle\rho^{2}\right\rangle\right) \mathcal{A}_{\vec{k}}=0
$$

where $\rho^{2}=2 \phi^{\dagger} \phi, \vec{k}$ is the comoving momentum, e electric charge $\left(e^{2} / 4 \pi \equiv \alpha \approx 1 / 137\right)$, and $\tau$ the conformal time, which is related to the "physical" time $t$ as $d t=a(\tau) d \tau$. The average $\langle\cdot\rangle$ should be computed in the Hartree approximation by subtracting the vacuum contribution, so that in the vacuum $\left\langle\rho^{2}\right\rangle \rightarrow 0$ and Eq. (2) reduces to that of a harmonic oscillator. The solutions are then travelling harmonic waves,

$$
\mathcal{A}_{\mathrm{vac}}^{( \pm)}=\left(\frac{2 \pi V}{k}\right)^{\frac{1}{2}} e^{\mp i k \tau}
$$


where $k=|\vec{k}|$ and we used the (Wronskian) normalisation of the mode functions, $\mathbf{W}\left[\mathcal{A}_{\mathrm{vac}}^{(+)}, \mathcal{A}_{\mathrm{vac}}^{(-)}\right]$ $=4 \pi i V$, appropriate for periodic boundary conditions in a box of volume $V$. The modulus of the mode functions in Eq. (3) does not depend on time, which is a consequence of conformal invariance being restored when $\left\langle\rho^{2}\right\rangle \rightarrow\left\langle\rho^{2}\right\rangle_{\text {vac }}=0$.

For simplicity we shall consider only homogeneous space-times such that the scale factor $a$ in Eq. (国) may be taken to be

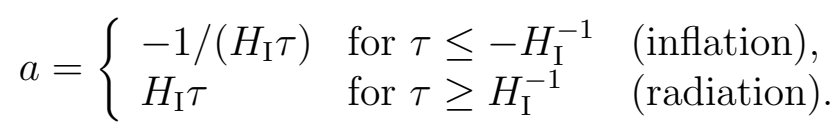

This corresponds to the following smooth matching at the inflation-radiation transition: $a\left(-H_{\mathrm{I}}^{-1}\right)=a\left(H_{\mathrm{I}}^{-1}\right)$ and $d a\left(-H_{\mathrm{I}}^{-1}\right) / d \tau=d a\left(H_{\mathrm{I}}^{-1}\right) / d \tau$.

\subsection{Breakdown of conformal invariance}

The backreaction term in Eq. (2) is generated during inflation because of the gravitational coupling of the scalar field. In the Hartree approximation, the backreaction is described by elastic scattering processes only, which should be a good approximation in inflation. We also assume that damping is negligible in inflation, since most of the amplification is on superhorizon scales beyond the reach of dissipative processes.

The Hartree term $\left\langle\rho^{2}\right\rangle$ for a light, charged scalar field can be estimated as follows. From the scalar equation of motion, one infers that during inflation the scalar field grows until its effective mass essentially reaches the Hubble parameter. More precisely, we have $m_{\phi}^{2}+$ $3 \lambda_{\phi}\left\langle\rho^{2}\right\rangle \leq 2 H_{\mathrm{I}}^{2}$, so that $e^{2}\left\langle\rho^{2}\right\rangle / H_{\mathrm{I}}^{2} \leq 2 e^{2} /\left(3 \lambda_{\phi}\right) \simeq 1 /\left(16 \lambda_{\phi}\right)$. When the limit is saturated, the growth of $\phi$ modes becomes exponentially suppressed. We assume that this occurs well before the end of inflation. The solutions of Eq. (2) may then be conveniently written in terms of Hankel functions as follows:

$$
\mathcal{A}_{\vec{k}}^{(i)}=\pi(-\tau V)^{\frac{1}{2}} H_{\nu}^{(i)}(-k \tau), \quad i=1,2, \quad \nu^{2}=\frac{1}{4}-\frac{e^{2}\left\langle\rho^{2}\right\rangle}{H_{\mathrm{I}}^{2}} \quad \text { (inflation). }
$$

For the mode-function normalisation we impose the Wronskian condition $\mathbf{W}\left[\mathcal{A}_{\vec{k}}^{(1)}, \mathcal{A}_{\vec{k}}^{(2)}\right]=$ $4 \pi i V$ so that, as $\tau \rightarrow-\infty$, the mode functions reduce to the vacuum mode functions discussed above: $\mathcal{A}_{\vec{k}}^{(1,2)} \rightarrow \mathcal{A}_{\mathrm{vac}}^{( \pm)}$. At later stages of inflation, however, the mode functions get "squeezed" on superhorizon scales as

$$
\mathcal{A}_{\vec{k}}^{(j)} \stackrel{k|\tau| \ll 1}{\longrightarrow} i(-1)^{j} \Gamma(\nu)\left(\frac{2 V}{k}\right)^{\frac{1}{2}}\left(-\frac{k \tau}{2}\right)^{\frac{1}{2}-\nu}, \quad j=1,2 .
$$


Since $\operatorname{Re}[\nu]<1 / 2$, the amplitudes $\mathcal{A}_{\vec{k}}^{(j)}$ actually decrease, while the momenta $\partial_{\tau} \mathcal{A}_{\vec{k}}^{(j)}$ grow (see Fig. 目). This evolution preserves the uncertainty relation for the photon field operators, which can be equivalently expressed by the (conserved) Wronskian.

After inflation the squeezed photon state evolves according to complicated physics associated with the processes of preheating and thermalisation of the Universe. The details of the evolution are model dependent and deserve further study [10], especially since these processes have not been fully considered in the context of gauge fields (see, however, Ref. [11]). In the following section we shall make a crude approximation and assume that the scalar field decays instantaneously at the end of inflation, leading to sudden restoration of conformal symmetry. The effects of preheating and thermalisation are considered in section @, where we show that, under certain conditions, preheating results in additional amplification of the superhorizon modes.

\section{Photon field amplification from inflation}

Here we assume that soon after inflation the $\phi$ field decays nonadiabatically such that $\left\langle\rho^{2}\right\rangle$ quickly approaches the vacuum value $\left\langle\rho^{2}\right\rangle_{\text {vac }}=0$, rendering Eq. (2) conformally invariant. The solutions in the radiation era are then linear combinations of the travelling waves $\mathcal{A}_{\text {vac }}^{( \pm)}$ of Eq. (3). Particle production occurs since the effective mass parameter undergoes a nonadiabatic change at the inflation-radiation transition. This change can be nonadiabatic even when the squared mass $e^{2}\left\langle\rho^{2}\right\rangle$ changes adiabatically. Indeed, at the beginning of radiation era the scale factor evolves nonadiabatically, i.e. $\left|\left(\partial_{\tau} a^{2}\right) / a^{2}\right|^{2}=4 H_{\mathrm{I}}^{2} \gg e^{2}\left\langle\rho^{2}\right\rangle$, so that the results concerning the photon field amplification in the simple case under consideration are quite generic.

Since the vacuum of inflation does not correspond to the late-time vacuum of the radiation era, the field content of Eq. (5) may be interpreted from the point of view of a late-time observer by a smooth matching of the mode functions $\mathcal{A}_{\vec{k}}^{(i)}$ at the end of inflation to a linear combination of travelling waves in the radiation era,

$$
\begin{aligned}
\mathcal{A}_{\vec{k}}^{(1)}\left(-H_{\mathrm{I}}^{-1}\right) & =\alpha_{\vec{k}} \mathcal{A}_{\vec{k}}^{(+)}\left(H_{\mathrm{I}}^{-1}\right)+\beta_{-\vec{k}}^{*} \mathcal{A}_{-\vec{k}}^{(-)}\left(H_{\mathrm{I}}^{-1}\right) \\
\partial_{\tau} \mathcal{A}_{\vec{k}}^{(1)}\left(-H_{\mathrm{I}}^{-1}\right) & =\alpha_{\vec{k}} \partial_{\tau} \mathcal{A}_{\vec{k}}^{(+)}\left(H_{\mathrm{I}}^{-1}\right)+\beta_{-\vec{k}}^{*} \partial_{\tau} \mathcal{A}_{-\vec{k}}^{(-)}\left(H_{\mathrm{I}}^{-1}\right)
\end{aligned}
$$

and similarly for $\mathcal{A}_{\vec{k}}^{(2)}=\mathcal{A}_{\vec{k}}^{(1) *}$. Here $\alpha_{\vec{k}}$ and $\beta_{\vec{k}}$ are the Bogoliubov coefficients that relate 


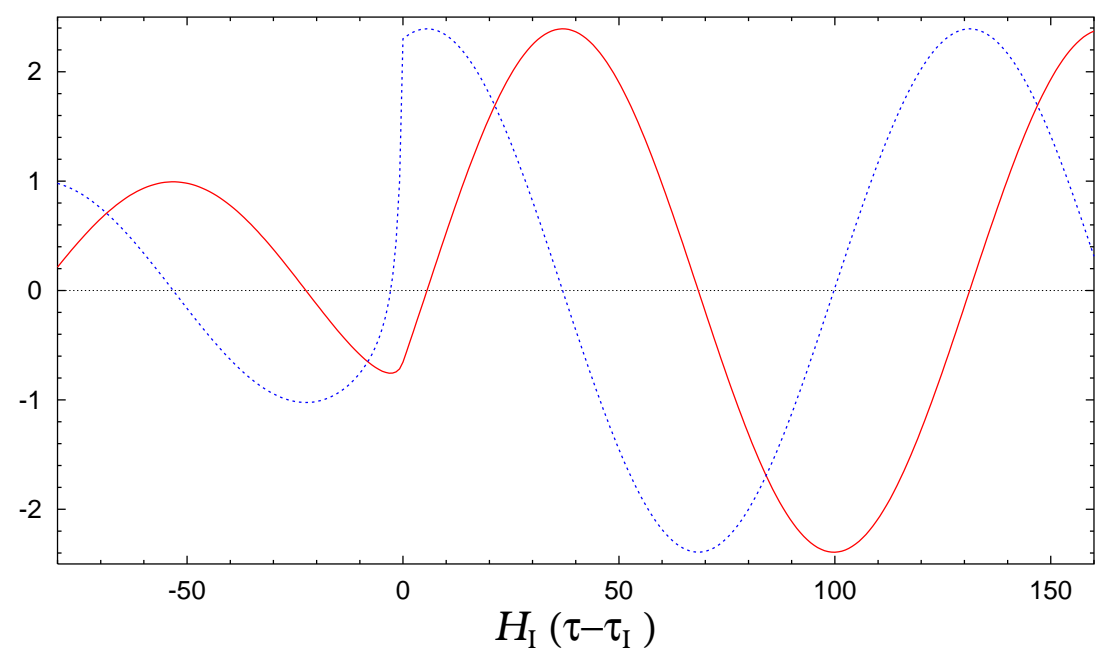

Figure 1: Evolution of (the imaginary part of) the gauge-field amplitude $\operatorname{Im}\left[\mathcal{A}_{\vec{k}}\right]$ (red solid curve) and its momentum $\operatorname{Im}\left[\partial_{\tau} \mathcal{A}_{\vec{k}}\right]$ (blue dotted curve) for $k / H_{\mathrm{I}}=0.05$ near the inflation-radiation transition, which occurs at $\tau=\tau_{\mathrm{I}}= \pm H_{\mathrm{I}}^{-1}$. As a consequence of broken conformal invariance, toward the end of inflation the amplitude decreases and the derivative increases, leading to an enhanced amplitude in the radiation era. The normalisation is chosen so that both functions would have amplitude equal to unity in the conformally evolving case, and we use $\nu=0.2$ for the purpose of illustration.

the creation and annihilation operators in the radiation era to those of (de Sitter) inflation.] With $\mathcal{A}_{\vec{k}}^{( \pm)}=\mathcal{A}_{\text {vac }}^{( \pm)}$the matching problem is easily solved. On superhorizon scales we have

$$
\left(\begin{array}{c}
\alpha_{\vec{k}} \\
\beta_{\vec{k}}
\end{array}\right)=\mp e^{i k / H_{\mathrm{I}}} \frac{\Gamma(\nu)}{4 \sqrt{\pi}}\left(\frac{1}{2}-\nu\right)\left(\frac{k}{2 H_{\mathrm{I}}}\right)^{-\frac{1}{2}-\nu}+\mathcal{O}\left(\left(k / 2 H_{\mathrm{I}}\right)^{-\frac{1}{2}+\nu}\right)
$$

so that at late time in radiation the linearly independent mode functions $\mathcal{A}_{\vec{k}}$ and $\mathcal{A}_{\vec{k}}{ }^{*}$, where $\mathcal{A}_{\vec{k}} \equiv \alpha_{\vec{k}} \mathcal{A}_{\vec{k}}^{(+)}+\beta_{-\vec{k}}^{*} \mathcal{A}_{-\vec{k}}^{(-)}$, are proportional to $k^{-1-\nu}$. When $\nu \approx 1 / 2$ the superhorizon modes $\mathcal{A}_{\vec{k}} \propto k^{-3 / 2}$ make up an almost scale-invariant spectrum, similar to that of a massless scalar field. The amplification by a factor $\beta_{\vec{k}} \propto\left(k / H_{\mathrm{I}}\right)^{-1 / 2-\nu}$ compared to the Minkowski vacuum spectrum (see Fig. 1) is a consequence of the large value of the momentum $\partial_{\tau} \mathcal{A}_{\vec{k}}^{(j)}$ at the end of inflation, as can be inferred from Eq. (6). This is the maximum amplification that can be attained at the inflation-radiation transition, since sudden transition is the one with maximal nonadiabaticity. However, it is not hard to show that a softer matching, for example onto

\footnotetext{
${ }^{1}$ The convention used here is consistent with the Bogoliubov coefficients being defined through the following relations: $a_{\vec{k}}=\alpha_{\vec{k}} \bar{a}_{\vec{k}}+\beta_{\vec{k}} \bar{b}_{-\vec{k}}^{\dagger}, b_{\vec{k}}=\alpha_{\vec{k}} \bar{b}_{\vec{k}}+\beta_{\vec{k}} \bar{a}_{-\vec{k}}^{\dagger}$, where barred operators are for inflation and unbarred for radiation era.
} 
$\left\langle\rho^{2}\right\rangle \sim H^{2}=\left(H_{\mathrm{I}} \tau^{2}\right)^{-2}$, leads to almost as strong an amplification on superhorizon scales (for $\nu \approx 1 / 2)$, namely $\mathcal{A}_{\vec{k}} \propto k^{-\frac{1}{2}-2 \nu}$. An obvious candidate for such a scenario is "gravitational" preheating [12]. We emphasise that, in a transition between asymptotic "in" and "out" states with the property $\left\langle\rho^{2}\right\rangle_{\text {in }}=\left\langle\rho^{2}\right\rangle_{\text {out }}=0$, the expectation value $\left\langle\rho^{2}\right\rangle$ will typically be non-zero during any period when the quantum operator $\rho^{2}$ evolves non-adiabatically, leading to amplification of gauge fields.

\section{Photon field amplification in preheating}

Here we present a simplified model of preheating in which the spectrum of photons from inflation is further amplified on superhorizon scales, so long as inelastic scattering and dissipation can be neglected. We assume that, at late stages when thermalisation takes place, the $\phi$ field decays nonadiabatically, imprinting the photon-field spectrum onto the plasma as described in section 3. How realistic this assumption is will be the subject of a separate study [10].

Resonant amplification of the photon field may result when the scalar field $\phi$ couples to the inflaton and grows resonantly such that the photon mass term $e^{2}\left\langle\varphi^{2}\right\rangle\left(\varphi^{2}=2 a^{2} \phi^{\dagger} \phi\right)$ acquires an oscillatory component, leading to (secondary) resonant amplification of the photon field $A$. This resonant amplification resembles stochastic resonance [13 in which all superhorizon modes are equally amplified. We shall now make a crude estimate of the amplification factor.

We assume massless chaotic inflation with a quartic interaction term $\lambda_{s} s^{4} / 4$, where $\lambda_{s} \simeq$ $10^{-13}$ as specified by the COBE satellite microwave background radiation measurements. Furthermore, the inflaton $s$ couples to $\phi$ with a term $h s^{2} \phi^{\dagger} \phi$ such that $h s^{2} \leq 2 H^{2}(s)$ during inflation, setting an upper limit on $h$. It is now easy to show that the oscillating inflaton $s$ decays into $\phi$ through a narrow resonance with the quality factor $q_{\phi} \approx h s_{0}^{2} / 4 \omega_{\mathrm{I}}^{2} \leq 1 / 200$, where $s_{0} \approx 0.3 M_{\mathrm{P}}$ is the inflaton amplitude at the end of inflation and $\omega_{\mathrm{I}}=c_{n} \sqrt{\lambda_{s}} s_{0}$ $\left(c_{n} \approx 0.847\right)$ is the inflaton frequency. Since at very early stages the photon-field quality factor $q_{A}=e^{2}\left\langle\varphi^{2}\right\rangle_{\text {osc }} / 4 \omega_{\mathrm{I}}^{2} \ll q_{\phi}$, where $\left\langle\varphi^{2}\right\rangle_{\text {osc }}$ is the amplitude of the oscillatory component of $\left\langle\varphi^{2}\right\rangle$, energy density $\rho$ is much more efficiently transferred from $s$ to $\phi$ than it is from $\phi$ to $A$. As time passes, $\left\langle\varphi^{2}\right\rangle_{\text {osc }}$ grows, leading to more efficient resonant production of the photon field $A$. Quite generically, the photon-field superhorizon modes begin to grow when $q_{A} \sim 1$ is attained [13]. At that time $\tau_{1}, \rho_{A}\left(\tau_{1}\right) \leq \rho_{\phi}\left(\tau_{1}\right) \sim 12 \lambda_{\phi} \omega_{\mathrm{I}}^{4} / e^{4}$. The modes stop growing 
when $\rho_{\phi} \sim \rho_{A} \sim \rho_{s} \simeq \omega_{\mathrm{I}}^{4} /\left(4 \lambda_{s} c_{n}^{4}\right)$. On the other hand, a resonating vector mode grows as $\mathcal{A}_{\vec{k}} \propto e^{\mu_{A} \omega_{\mathrm{I}} \tau}$ and hence $\rho_{A} \propto e^{2 \mu_{A} \omega_{\mathrm{I}} \tau}$, implying the following estimate of the amplification factor $\chi$ for superhorizon modes during preheating

$$
\chi \sim\left(\frac{\rho_{s}}{\rho_{A}\left(\tau_{1}\right)}\right)^{\frac{1}{2}} \sim \frac{e^{2}}{4 c_{n}^{2}} \frac{1}{\sqrt{3 \lambda_{\phi} \lambda_{s}}} \sim 10^{5}
$$

We assume that the photon field at this point freezes in due to large conductivity. The purpose of this simple analysis was to show that superhorizon modes are amplified during preheating, a rough estimate of the amplification factor being Eq. (9). To obtain a more accurate value, the problem should be reanalysed using numerical techniques. We have also studied the evolution of the spectrum by matching the inflation mode functions to those in preheating, $\mathcal{A}_{\text {preh }}^{( \pm)}=\left(2 \pi V / \omega_{\mathrm{I}}\right)^{1 / 2} e^{\left(\mu_{A} \mp i\right) \omega_{I} \tau}$. At the end of preheating, the spectrum is $\mathcal{A}_{\text {preh }} \propto k^{-\nu}$, as in Eq. (6), with an additional amplification factor given by Eq. (9). A final matching of the preheating photon modes onto the massless photon modes in radiation results in the spectrum $\mathcal{A}_{\text {rad }} \propto k^{-1-\nu}$ computed in section 3 , again with the additional amplification factor (9).

\section{$5 \quad$ Magnetic field production in the standard model}

In this section we show that the amplification mechanism, which we illustrated with the example of scalar electrodynamics, is operative for the standard-model $Z$ field just as it is for any gauge field that couples to a light scalar field. Namely, in the $Z$-field mode equation (cf. Eq. (2))

$$
\left(\partial_{\tau}^{2}+\vec{k}^{2}+\frac{m_{Z}^{2}}{v^{2}} a^{2}\left\langle\rho^{2}\right\rangle\right) \mathcal{Z}_{\vec{k}}=0
$$

conformal invariance is broken by the Hartree backreaction term of the standard-model Higgs field $\Phi$. Here $v=246 \mathrm{GeV}$ and $\rho^{2}=2 \Phi^{\dagger} \Phi$. The restriction $3 \lambda_{H}\left\langle\rho^{2}\right\rangle \leq 2 H_{\mathrm{I}}^{2} \mathrm{im}$ plies that, in order to obtain substantial amplification of superhorizon modes $(\nu \gtrsim 1 / 3)$, we require $\lambda_{H} \gtrsim 0.66$. Equivalently, the Higgs-boson mass must satisfy the lower bound $m_{H}=v \sqrt{2 \lambda_{H}} \gtrsim 280 \mathrm{GeV}$. The spectrum reads

$$
\mathcal{Z}_{\vec{k}} \equiv \alpha_{\vec{k}} \mathcal{Z}_{\vec{k}}^{(+)}+\beta_{-\vec{k}}^{*} \mathcal{Z}_{-\vec{k}}^{(-)} \propto k^{-1-\nu} \quad \text { with } \quad \nu^{2}=\frac{1}{4}-\frac{m_{Z}^{2}}{v^{2}} \frac{\left\langle\rho^{2}\right\rangle}{H_{\mathrm{I}}^{2}}
$$




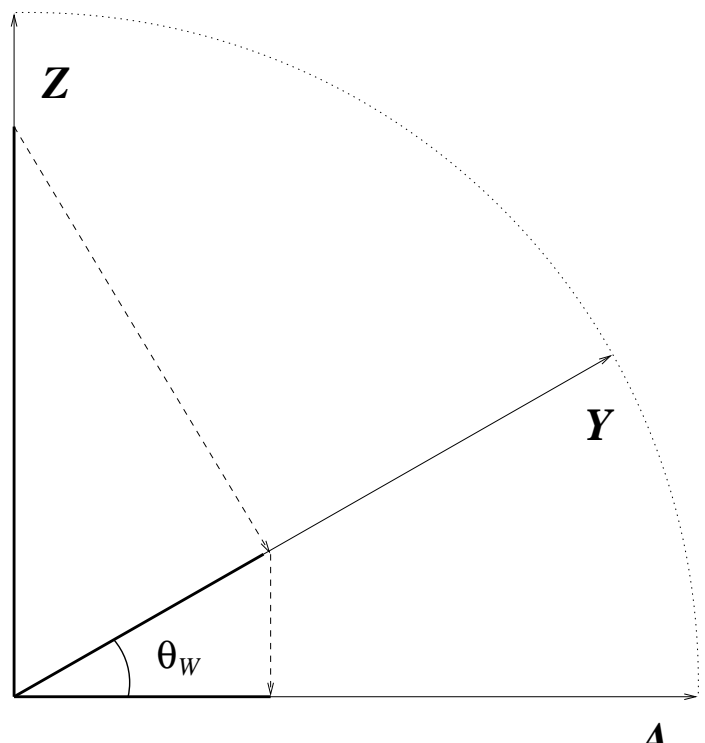

Figure 2: Projection of the primordial spectrum of the standard-model $Z$ field first onto the hypercharge field $Y$ (at the inflation-radiation transition) and then onto the photon field $A$ (at the electroweak phase transition).

where $\mathcal{Z}_{\vec{k}}^{( \pm)} \equiv \mathcal{Z}_{\text {vac }}^{( \pm)}=(2 \pi V / k)^{1 / 2} e^{\mp i k \tau}$ (cf. Eq. (3i)), and $\alpha_{\vec{k}}$ and $\beta_{\vec{k}}$ are given in Eq. (8)). The non-Abelian component of the $Z$ field, i.e. $W^{(3)}$, develops a magnetic mass and becomes screened as the Universe thermalises. Only its Abelian component, the hypercharge field $Y=$ $-\sin \theta_{\mathrm{W}} Z$, survives and freezes into the plasma. Here $\theta_{\mathrm{W}}$ is the Weinberg angle $\left(\sin \theta_{\mathrm{W}} \approx\right.$ 0.23). After the electroweak transition it is the photon field which remains unscreened with amplitude $A=\cos \theta_{\mathrm{W}} Y$. Note that, although the $Z$ field and the photon field are orthogonal, the resulting photon spectrum is identical to that of the original $Z$ field, apart from an amplitude suppression factor $\sin \theta_{\mathrm{W}} \cos \theta_{\mathrm{W}} \approx 0.42$.

The situation is analogous to that of light polarisers (see Fig. 2). With two orthogonal light polarisers no light passes. When a third polariser is inserted at an angle $\theta$ (with respect to the second polariser) however, some of the photons do pass. The photon amplitude is reduced by $\sin \theta \cos \theta$, just as in the Z-field case. The main advantage of the amplification mechanism presented here is its naturalness. Indeed, no fields are required except those of the standard model and inflation.

One should also consider the possibility of additional enhancement of the field $Y=$ $-\sin \theta_{\mathrm{W}} Z$ on superhorizon scales during preheating. For this purpose, we envision a hybrid- 
inflation model in which inflation is driven by the vacuum energy of a GUT Higgs field. After inflation ends, this field begins to oscillate, leading to resonant production of some superheavy gauge fields $X_{i}$. As a consequence the hypercharge field $Y$, which couples to the $X_{i}$, grows as well, and freezes in when the Universe thermalises. The details of this amplification mechanism are model-specific and will be presented elsewhere [10]. Finally, we emphasise that the superheavy gauge fields grow resonantly during preheating and suddenly decay after preheating when they become supermassive. These are required properties of the scalar field in the scalar-electrodynamics model considered in sections 2 and 3 .

\section{Magnetic field spectrum}

We now consider the possibility that primordial gauge fields from inflation can provide the necessary seed for the galactic dynamo mechanism [8] and thereby explain the observed presence of micro-Gauss-strength magnetic fields in a large number of spiral galaxies [3].

The dynamo mechanism amplifies a weak, coherent seed field using the differential rotation of a galaxy in conjunction with the turbulent motion of ionised gas. The length scale relevant for the operation of the galactic dynamo is the size of the largest turbulent eddy, $\sim 100$ pc. On this scale, there is a minimum strength of the magnetic seed field for which the dynamo can operate. Estimates of this minimal strength lie in the range $10^{-23}-10^{-19} \mathrm{G}$ for a universe with critical matter density and zero cosmological constant. The lower bound can be relaxed [14 to about $10^{-30} \mathrm{G}$ for a flat, low-density universe with a dark-energy component (e.g. a cosmological constant or quintessence), which appears to be favoured by recent results from supernova observations and balloon experiments [15, 16].

The dynamo scale $(100 \mathrm{pc})$ corresponds to a comoving scale today of $\ell_{c} \sim 10 \mathrm{kpc} \approx$ $1.56 \times 10^{36} \mathrm{GeV}^{-1}$ before gravitational collapse and galaxy formation [14. Because the concentration of matter into a galaxy brings about an amplification of magnetic fields by a factor $\left(\rho_{\text {gal }} / \rho_{0}\right)^{2 / 3} \approx 5 \times 10^{3}$, the bounds that should be imposed on the scale $\ell_{c}$ are $B_{\text {seed }} \gtrsim 2 \times 10^{-27} \mathrm{G}$ for a universe with critical matter density and $B_{\text {seed }} \gtrsim 2 \times 10^{-34} \mathrm{G}$ for a flat, dark-energy dominated, low-density universe.

We consider first the spectrum resulting from scalar electrodynamics (section 3) and the $Z$-field case (section 5) and neglect for the time being further amplification which may result from parametric resonance during preheating. At the end of inflation, the scale factor 
corresponds to an equivalent temperature $T$ given by $H_{\mathrm{I}}=\pi g_{*}^{1 / 2}(T) T^{2} /\left(\sqrt{90} M_{\mathrm{P}}\right)$, where $g_{*}(T) \sim 10^{2}$ is the number of relativistic degrees of freedom. Assuming first that the primordial magnetic field is frozen in the plasma from the time of its creation, the relevant length scale for the magnetic field at the end of inflation is $\ell=\ell_{\mathrm{c}} T_{0} / T$, where $T_{0} \approx 2.73 \mathrm{~K}$ is the temperature today.

For $\bar{k} \equiv 2 \pi / \ell \ll H_{\mathrm{I}}$, the Bogoliubov coefficients $\alpha_{\vec{k}}$ and $\beta_{\vec{k}}$ given by Eq. (8) are maximal for $\nu=\bar{\nu} \approx 1 / 2-1 / \ln \left(2 H_{\mathrm{I}} / \bar{k}\right)$. For this optimal value of $\nu$ one obtains the mode function

$$
\left|\mathcal{A}_{\vec{k}}\right| \sim \frac{1}{4} \Gamma(\bar{\nu})\left(\frac{1}{2}-\bar{\nu}\right)\left(\frac{V}{H_{\mathrm{I}}}\right)^{\frac{1}{2}}\left(\frac{k}{2 H_{\mathrm{I}}}\right)^{-\bar{\nu}-1} .
$$

The magnetic field correlated on a particular scale $\ell$ is defined by

$$
\begin{aligned}
& B_{\ell}^{2}=\left\langle B_{i}(\ell, \vec{x}) B_{i}(\ell, \vec{x})\right\rangle-\left\langle B_{i}(\ell, \vec{x}) B_{i}(\ell, \vec{x})\right\rangle_{\mathrm{vac}}, \\
& B_{i}(\ell, \vec{x})=\frac{3}{4 \pi \ell^{3}} \int_{|\vec{y}-\vec{x}| \leq \ell} d^{3} y B_{i}(\vec{y}),
\end{aligned}
$$

where the average $\langle\cdot\rangle$ is taken over Fock space as well as the position $\vec{x}$. For $\ell H_{\mathrm{I}} \gg 1$ one finds

$$
B_{\ell}=3 \times 2^{\bar{\nu}-2} \frac{\Gamma(\bar{\nu})}{\pi}\left(\frac{1}{2}-\bar{\nu}\right) H_{\mathrm{I}}^{2}\left(\ell H_{\mathrm{I}}\right)^{\bar{\nu}-\frac{3}{2}} .
$$

The magnetic field then is assumed to be frozen in the plasma such that today $B_{\ell_{c}}=$ $B_{\ell}\left(T_{0} / T\right)^{2}$. Since roughly $B_{\ell_{\mathrm{c}}} \propto T$, both $B_{\ell_{\mathrm{c}}}$ and $B_{\ell}$ are larger for higher inflation scale $H_{\mathrm{I}} \propto T^{2}$. Taking $H_{\mathrm{I}} \sim 10^{13} \mathrm{GeV}$ (corresponding to $T \sim 10^{15} \mathrm{GeV}$ ) we find $B_{\ell} \sim 10^{22} \mathrm{G}$ at the end of inflation, and $B_{\ell_{\mathrm{c}}} \sim 10^{-34} \mathrm{G}$ today at a comoving scale of $10 \mathrm{kpc}$ (see Fig. (3)).

In section 1 we showed that this field strength can be further amplified by a factor of about $10^{5}$ through parametric resonance with an oscillating scalar field, leading to $B_{\ell_{c}} \sim 10^{-29} \mathrm{G}$. A supplementary increase in field strength is obtained if we assume that the magnetic field does not freeze into the plasma upon creation, but rather that its correlation length grows quicker than the scale factor, as is the case for helical turbulence [17]. Such a causal mechanism can only operate on a given comoving scale after this scale has reentered the horizon. One can show that the growth of correlations due to turbulent evolution leads to an additional amplification of about $\left(\ell_{\mathrm{eq}} / \ell_{\mathrm{c}}\right)^{2(1-\nu) / 3}$, where $\ell_{\mathrm{eq}} \sim 50 \mathrm{Mpc}$ denotes the equal matter-radiation horizon today. For $\ell_{\mathrm{c}}=10 \mathrm{kpc}$ the amplification is about 20. For other types of turbulence the amplification may be somewhat smaller. 


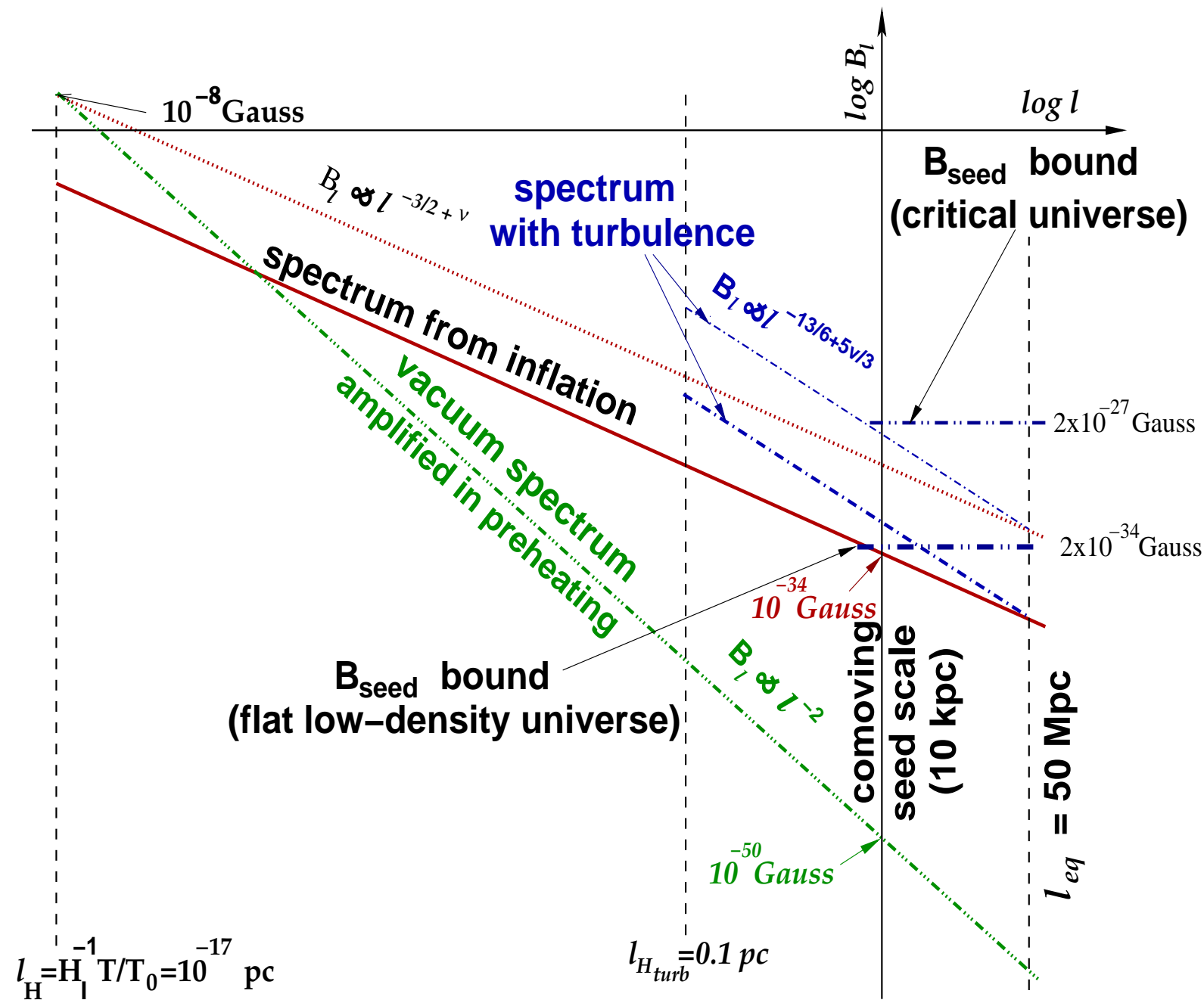

Figure 3: Magnetic-field spectra and relevant seed-field bounds. In green (dash-dot-dot-dot) we show the vacuum spectrum $B_{\ell} \propto \ell^{-2}$ obtained from preheating, assuming an amplification factor of $10^{5}$. At the comoving scale $\ell_{c} \sim 10 \mathrm{kpc}, B_{\ell_{c}} \sim 10^{-50} \mathrm{G}$. In red (dots and solid) the spectrum $B_{\ell} \propto \ell^{-3 / 2+\nu}$ from inflation in our mechanism is shown, with and without preheating amplification. For this spectrum, $B_{\ell_{c}} \sim 10^{-29} \mathrm{G}$ and $B_{\ell_{c}} \sim 10^{-34} \mathrm{G}$, respectively. We also show (blue dashdots) the spectrum enhanced by helical turbulence (at $\ell_{c} \sim 10 \mathrm{kpc}$ an enhancement of about 20 is obtained). This is to be compared with the dynamo bounds rescaled by a factor $5 \times 10^{3}$ (see main text) $B_{\text {seed }} \gtrsim 2 \times 10^{-27} \mathrm{G}$ for a universe with critical matter density, and $B_{\text {seed }} \gtrsim 2 \times 10^{-34} \mathrm{G}$ for a flat, low-density universe. 
In Fig. 3 we display the spectrum $B_{\ell}$ of the magnetic field for the different histories of amplification discussed in this section and compare with the comoving $\ell_{\mathrm{c}}=10 \mathrm{kpc}$ seed-field bounds, which are $B_{\text {seed }} \gtrsim 2 \times 10^{-27} \mathrm{G}$ in a universe with critical matter density (presently disfavoured by observations) and $B_{\text {seed }} \gtrsim 2 \times 10^{-34} \mathrm{G}$ in a flat, low-density universe dominated by dark energy. The corresponding field strengths and bounds in a newborn galaxy can be obtained by multiplying with a factor $5 \times 10^{3}$.

To conclude, in this Letter we have presented a generic mechanism for production of gauge fields during inflation which predicts an almost scale-invariant spectrum for gauge fields, comparable with the scale-invariant spectrum of cosmological perturbations. When applied to electromagnetism, the resulting magnetic-field spectrum $B_{\ell} \propto \ell^{\nu-3 / 2} \sim \ell^{-1}$ can provide strong enough seed fields to explain the origin of galactic magnetic fields.

\section{Acknowledgements}

TP wishes to thank M. Giovannini and M. Shaposhnikov for useful discussions. Support for KD was provided by DGICYT grant PB98-0693 and by the European Union under contract ERBFMRX-CT96-0090; for OT by the European Union under contract ERBFMBI-CT972697 and by a CERN visiting fellowship. We gratefully acknowledge travel support by the U.K. PPARC.

\section{References}

[1] V.F. Mukhanov and G.V. Chibisov, Pisma Zh. Eksp. Teor. Fiz. 33, 549 (1981) [JETP Lett. 33, 532 (1981)].

[2] S.W. Hawking, Phys. Lett. B115, 295 (1982).

[3] P.P. Kronberg, Rept. Prog. Phys. 57, 325 (1994).

[4] J. Adams, U.H. Danielsson, D. Grasso, and H. Rubinstein, Phys. Lett. B388, 253 (1996); John D. Barrow, Pedro G. Ferreira and Joseph Silk, Phys. Rev. Lett. 78, 3610 (1997), astro-ph/9701063; A. Kosowsky and A. Loeb, Ap. J. 469, 1 (1996); R. Durrer, P.G. Ferreira, and T. Kahniashvili, Phys. Rev. D 61, 043001 (2000), astro-ph/9911040.

[5] A.D. Dolgov, Zh. Eksp. Teor. Fiz. 81, 417 (1981) [Sov. Phys. JETP 54(2), 223 (1981)]; Phys. Rev. D 48, 2499 (1993). 
[6] M. Turner and L.M. Widrow, Phys. Rev. D 37, 2743 (1987).

[7] E.A. Calzetta, A. Kandus, and F.D. Mazzitelli, Phys. Rev. D 57, 7139 (1998), astroph/9707220; A. Kandus, E.A. Calzetta, F.D. Mazzitelli, and C.E.M. Wagner, Phys. Lett. B472, 287 (2000), hep-ph/9908524.

[8] E.N. Parker, Ap. J. 162, 665 (1970); ibid. 163, 255 (1971); ibid. 279, (1971); E.N. Parker, Cosmic magnetic fields (Clarendon, Oxford, England, 1979); Ya.B. Zeldovich, A.A. Ruzmaikin and D.D. Sokoloff, Magnetic fields in astrophysics (Gordon and Breach, New York, 1983).

[9] M. Giovannini and M. Shaposhnikov, hep-ph/0004269.

[10] A.-C. Davis, K. Dimopoulos, T. Prokopec and O. Törnkvist, in progress.

[11] J. Baacke, K. Heitmann and C. Patzold, Phys. Rev. D 55, 7815 (1997), hep-ph/9612264; R. Brustein and D.H. Oaknin, Phys. Rev. D 60, 023508 (1999), hep-ph/9901242; F. Finelli and A. Gruppuso, hep-ph/0001231.

[12] L.H. Ford, Phys. Rev. D 35, 2955 (1987); B. Spokoiny, Phys. Lett. B315, 40 (1993), gr-qc/9306008; M. Joyce, Phys. Rev. D 55, 1875 (1997), hep-ph/9606223; M. Joyce and T. Prokopec, Phys. Rev. D 57, 6022 (1998), hep-ph/9709320; P.J.E. Peebles and A. Vilenkin, Phys. Rev. D 59, 063505 (1999), astro-ph/9810509.

[13] T. Prokopec and T.G. Roos, Phys. Rev. D 55, 3768 (1997), hep-ph/9610400; L. Kofman, A. Linde and A.A. Starobinsky, Phys. Rev. D 56, 3258 (1997), hep-ph/9704452.

[14] A.-C. Davis, M. Lilley and O. Törnkvist, Phys. Rev. D 60, 021301 (1999), astroph/9904022.

[15] S. Perlmutter et al., Ap. J. 517, 565P (1999); P.M. Garnavich et al., ibid. 509, 74G (1998).

[16] P. de Bernardis et al., Nature 404, 955 (2000), astro-ph/0004404; A. Balbi et al., astroph/0005124.

[17] D.T. Son, Phys. Rev. D 59, 063008 (1999); G.B. Field and S.M. Carroll, astroph/9811206. 